\title{
Experiencias ludocreativas en niñas y niños de quinto y sexto grado de Costa Rica: una forma innovadora de sentir los espacios educativos
}

\section{Ludo-creative experiences in girls and boys of fifth and sixth grade in Costa Rica: an innovative way of feeling educational spaces}

\author{
Mary Salazar Hernández*, Cristopher Montero Corrales** \\ *Universidad Técnica Nacional, Costa Rica. Contacto: msalazarh@utn.ac.cr \\ ** Universidad Técnica Nacional, Costa Rica. Contacto: cmontero@utn.ac.cr
}

Referencia/ reference:

Salazar, M. y Montero C. (2020). Experiencias ludocreativas en niñas y niños de quinto y sexto grado de Costa Rica: una forma innovadora de sentir los espacios educativos. Yulök Revista de Innovación Académica. 4(1), 65-80

\section{Resumen}

Este artículo reflexiona sobre los sentires de niñas y niños de quinto y sexto grado, de la escuela Bernardo Soto Alfaro, ubicada en la provincia de Alajuela y la escuela Mixta San Juan, situada en la provincia San José, capital de nuestro país. Es una sistematización de la experiencia llevada a cabo en talleres organizados de manera lúdica para la resolución pacífica de conflictos y el establecimiento de normas de compañerismo, impartidos por la Subcomisión Lúdico Creativa del Consejo Nacional de Rectores en el año 2019. Para llevar a cabo el objetivo del taller, se planifican metodologías lúdicas y creativas que fomentan la imaginación, la ternura, la esperanza y la espontaneidad, enfocados en ambientes de aprendizaje para la convivencia y la autotrascendencia.

Palabras clave: Lúdica, creatividad, infancia, autotrascendencia, convivencia.

\section{Abstract}

This article reflects on the feelings of fifth and sixth grade girls and boys from the Escuela Bernardo Soto Alfaro (School) located in the province of Alajuela; as well as ones of the Escuela Mixta San Juan (School), located in the San José province. It is a systematization of the experience carried out in workshops organized in a playful way for a peaceful resolution of conflicts and the establishment of companionship norms, taught by the Creative Playful Subcommittee of the National Council of Rectors in 2019 (Subcomisión Lúdico Creativa del Consejo Nacional de Rectores). To carry out the objective of the workshop, playful and creative methodologies are planned that promote imagination, tenderness, hope, and spontaneity focused on learning environments for coexistence and self-transcendence.

Keywords: Playful, creativity, childhood, self-transcendence, coexistence. 


\section{¿Por qué es importante sistematizar en Extensión y Acción Social?}

La sistematización de las experiencias entre la Universidad y la Sociedad transforma el ejercicio de la Docencia, la Investigación y la Extensión y Acción Social, por lo que implica una reflexión profunda de las maneras en que las personas académicas nos relacionamos con los miembros de las comunidades.

Incluso, algunas veces nos relacionamos, con actores sociales o en zonas geográficas con las cuales nos identificamos emocional e intelectualmente, ya sea, por historia de vida, afinidad o intereses personales.

Sistematizar no solo permite la generación y gestión del conocimiento científico, por medio de la producción académica como son artículos, informes, toma de decisiones institucionales, entre otros; sino que, también, tiene el potencial para hacer una experiencia significativa que propicie la mejora en la calidad de vida de las personas y de las comunidades.

Al respecto de lo anterior, Fantova (2003) aporta lo siguiente:

Quizá en la tradición y la realidad de la sistematización encontramos una forma de gestión del conocimiento especialmente atenta a lo relacional y lo participativo, a lo humano y lo popular, a lo informal y lo cultural, frente a versiones más frías, virtuales, informatizadas o economicistas (valgan los epítetos para entendernos) del asunto. (p.10)

La posibilidad de innovar procesos y prácticas académicas en beneficio de la pertinencia de la educación superior es una de las maneras en que la sistematización puede ser beneficiosa, ya que, promueve la dinámica relacional entre Docencia, Investigación y Extensión y Acción Social.

Es decir, por un lado, que la Universidad mejore sus procesos internos para seguir construyéndose como un agente radical de bienestar y educación. Como afirma Sánchez (2010): "la sistematización de experiencias está vinculada con la metodología de Investigación Acción Participativa (IAP), dado que una de sus características es que, a partir de reflexiones colectivas, los involucrados se autoinvestigan para comprender y generar activamente cambios institucionales y/o sociales" (p. 5).

Como indica el párrafo anterior, la sistematización en Extensión y Acción Social posibilita cambios instituciona- les, la mejora de nuestras maneras de vincularnos, como académicas y académicos con las comunidades y cambios sociales que repercutirían para colaborar de mejor manera con el bienestar de las personas.

Los productos de divulgación de la sistematización de las experiencias son formas para generar conocimiento científico validado, los cuales enriquecen las vivencias de la comunidad estudiantil, del personal académico, administrativo y de la sociedad. Son narrativas que intentan, como afirma Palma (1992) que permiten "comprender y tratar con lo cualitativo en la realidad” (p. 9).

A la vez, se resaltan los saberes cotidianos de las comunidades y con acompañamiento académico podrían, incluso, ser parte de los conocimientos que se difunden en las Universidades. Conocimiento que se ha reflexionado a partir de la experiencia, como afirma Jara (2012) a continuación:

La experiencia es siempre vivencial: implica una vinculación física, emocional y también intelectual con el conjunto de elementos del entramado inmediato con el que me relaciono. Las experiencias son, por tanto, lugares vivos de creación y producción de saberes. Estos saberes cotidianos que poseemos todas las personas, forman parte de la experiencia. (p. 69).

La producción académica de las Universidades, como es la escritura de un artículo de sistematización de las experiencias, se convierte en una forma de expresión que beneficia las reflexiones en torno a la relación Universidad-Sociedad, tanto para los procesos, prácticas y procedimientos con que cuentan la Docencia, Investigación y Extensión y Acción Social, como a las personas académicas, las cuales tienen la posibilidad de desarrollarse profesionalmente.

Para la Universidad Técnica Nacional puede ser un camino para generar conocimiento científico tras once años de experiencia con las comunidades y, de esta manera, internalizar como institución los aprendizajes que se construyen con los actores sociales. Herramienta que aumentaría nuestros índices de investigación publicada. Así que, podemos entenderla, como una innovación con altas posibilidades de impacto en el quehacer universitario.

Si como afirma Boaventura de Sousa Santos, las universidades públicas se encuentran en una crisis de legitimidad, por lo tanto, reflexionar sobre las maneras en las que 
se construye la relación Universidad-Sociedad es vital porque permitiría reconquistar esa legitimidad, ya que "Los intereses sociales están articulados con los intereses científicos de los investigadores y la producción del conocimiento científico se da estrechamente ligada a la satisfacción de necesidades de los grupos sociales que no tienen el poder para el conocimiento técnico y especializado a través de la vía mercantil" (Sousa, 2007, P.66).

Como podemos ver, en el contexto actual de deslegitimación y mercantilización de la educación superior, el sistematizar experiencias de Extensión y Acción Social resulta ser una acción transformadora en tanto permite articular y enriquecer, la Docencia, la Investigación y la sensibilización social, como una actividad sustantiva y vital para la legitimidad de la vida universitaria.

\section{¿Qué vamos a sistematizar en este artículo?}

Sistematizaremos dos de las experiencias de talleres lúdico creativos realizadas por la Universidad Técnica Nacional (UTN), y de manera específica en la Subcomisión Lúdico Creativa del Consejo Nacional de Rectores (CONARE) en el año 2019, cuyo propósito se dirigía al fortalecimiento de los vínculos, las relaciones y las redes mediante experiencias ludocreativas en y entre las cinco universidades públicas y el enlace con organizaciones nacionales e internacionales (Subcomisión Lúdico Creativa, 2016).

En el año 2007, surge el proyecto "Talleres Lúdico Creativos", con representantes de la Universidad de Costa Rica (UCR), la Universidad Nacional (UNA), el Instituto Tecnológico de Costa Rica (ITCR) y la Universidad Estatal a Distancia (UNED). Posteriormente, en el año 2010, gracias a la determinación de la Comisión de Vicerrectores de Extensión y Acción Social, se transforma de proyecto a subcomisión ${ }^{1}$. Es hasta el año 2015, cuando la UTN se incorpora a la subcomisión participando como integrante hasta diciembre del 2019, al darse su cierre administrativo.
Con el cese de actividades de la subcomisión, se aumenta la necesidad y pertinencia de sistematizar sus experiencias y, de reflexionar, de manera crítica y participativa con las personas que se desempeñaron en las diferentes actividades que llevó a cabo, por medio de las cinco universidades públicas, desde los años 2007 hasta el 2019. Cabe señalar que durante ese periodo participaron 29289 personas, según los datos registrados en diversos informes de trabajo de la Subcomisión (Informe quinquenal de los años 2007 al 2015, 2016; Informe anual, 2016; Informe anual, 2017; Informe anual, 2018 e Informe anual, 2019 de la Subcomisión Lúdico Creativa).

En este artículo se dará énfasis a la reflexión de dos experiencias lúdico creativas denominadas: “ $¡ S o y$ creativ@ y resuelvo mis conflictos!" y "Amistad y Esperanza: un paso al futuro". Las cuales se llevaron a cabo con la participación de 60 personas: estudiantes de la educación general básica costarricense, de quinto y sexto grado en la escuela Mixta San Juan en San José y en la escuela Bernardo Soto Alfaro en Alajuela. Cabe destacar que estas se efectuaron como parte de las actividades que la Subcomisión organizó y planificó durante los años indicados anteriormente.

De las 60 personas inscritas en los talleres, 59 completaron el instrumento de evaluación que debían llenar al concluir la actividad, por lo que sus opiniones se convirtieron en insumos relevantes para la elaboración de este artículo.

A continuación, en las tablas 1 y 2 , se presenta la distribución por género y edad de las personas participantes:

1 Las subcomisiones de la Comisión de Vicerrectores de Extensión y Acción Social del Consejo Nacional de Rectores son entes oficiales que se han creado con la finalidad de "contribuir de forma colectiva al mejoramiento de la calidad de vida de poblaciones vulnerables y a la atención de temas estratégicos para el desarrollo del país" (Consejo Nacional de Rectores, 2017, p.1). A diferencia de los proyectos que deben definir un principio y final para ser aprobados en CONARE, las subcomisiones se distinguen por su durabilidad y su aprobación de planes anuales. Lo anterior refleja la importancia de las subcomisiones, ya que mediante el trabajo interuniversitario que se realiza en las mismas, según Moraga (2011) en Consejo Nacional de Rectores (2017), se genera un "Ligamen directo entre la universidad, el entorno y las realidades sociales, además, pone al servicio la capacidad académica institucional, con el propósito de lograr, en conjunto, las transformaciones requeridas para el mejoramiento de la calidad de vida de las personas" (p. 1). 
Tabla 1. Datos de la población escuela Bernardo Soto (sexto Grado).

\begin{tabular}{|l|c|c|c|c|c|}
\hline \multicolumn{1}{|c|}{ Población } & $\mathbf{1 1}$ años & $\mathbf{1 2}$ años & $\mathbf{1 3}$ años & $\mathbf{1 4}$ años & Total \\
\hline Femenino & 3 & 21 & 1 & 0 & 25 \\
\hline Masculino & 2 & 11 & 1 & 1 & 15 \\
\hline
\end{tabular}

Fuente: elaboración propia, 2019.

Tabla 2. Datos de la población escuela Mixta San Juan (quinto grado).

\begin{tabular}{|l|c|c|c|c|c|c|}
\hline \multicolumn{1}{|c|}{ Población } & $\mathbf{1 0}$ años & $\mathbf{1 1}$ años & $\mathbf{1 2}$ años & $\mathbf{1 3}$ años & $\mathbf{1 4}$ años & Total \\
\hline Femenino & 0 & 10 & 1 & 0 & 0 & 11 \\
\hline Masculino & 2 & 7 & 0 & 0 & 0 & 9 \\
\hline
\end{tabular}

Fuente: elaboración propia, 2019.

Los talleres lúdico creativos se realizaron en dos espacios distintos, el de la escuela Bernardo Soto se llevó a cabo en una quinta ubicada en El Coyol de Alajuela, con una duración aproximada de cinco horas y en el marco de la convivencia escolar de fin de año y, el de la escuela Mixta San Juan, con una duración aproximada de tres horas, se organizó en un aula del centro educativo, ubicado en Puriscal en San José. Ambos talleres se planificaron según el siguiente objetivo principal en común:

Que el estudiantado fortalezca la habilidad para la resolución pacífica de conflictos interpersonales que mejoren los vínculos de amistad y compañerismo en el aula, mediante la lúdica y la creatividad.

Es importante evidenciar la estructura general de los talleres lúdico creativos, que sirven de marco para explicitar y compartir la metodología utilizada en estas experiencias. Partiendo del hecho de que la ludocreatividad se aleja del tergiversado concepto "de juego y diversión como forma y no como contenido" (p.57), al cual hace referencia Pérez-Yglesias (2010), quien además manifiesta "Lo lúdico-creativo mal entendido como actividad -superficial y "light"- exclusivamente dedicada al ocio y entretenimiento se hermana e identifica con esta inadecuada noción de la acción social.” (p.57).

\section{Descripción metodológica de los talleres lúdi- co-creativos $^{2}$}

Tan importante como adquirir es sentir los conocimientos Jesús Paredes

Describir la estructura metodológica de los talleres lúdico-creativos llevados a cabo, nos brinda la oportunidad de acercarnos al concepto de lúdica, ya que, usualmente suele reducirse "a juego" y a una vivencia exclusiva para la etapa de la niñez. Sin embargo, la lúdica va más allá del juego, Prieto y Naranjo (2005), afirman que existen muchas concepciones equivocadas con respecto a la lúdica, entre ellas lo siguiente:

(...) la identificación con el juego, la recreación, el ocio y el tiempo libre. En ese sentido, es necesario aclarar que la lúdica no se restringe únicamente al juego, aunque no la desconoce, aquella es más profunda que el simple acto de jugar. (p. 310).

La lúdica es entendida como una dimensión inherente al ser humano y una actitud ante la vida, la cual se manifiesta de múltiples formas en diferentes espacios y etapas del ciclo vital. Como lo afirma Jiménez (2005) a continuación:

La lúdica como experiencia cultural es una actitud, una predisposición del ser frente a la cotidianidad, es una for-

2 Para el diseño y ejecución de los talleres lúdicos mencionados en este artículo se contó con la colaboración de la Licenciada en Trabajo Social Raquel Villafuerte Vega y la Licenciada en Trabajo Social Susana Ureña Mora, ambas miembros del equipo de apoyo Socioemocional del Proyecto Empléate del Programa de Desarrollo Empresarial de la Dirección de Extensión y Acción Social de la Universidad Técnica Nacional, quienes también han apoyado en otras actividades de la Subcomisión Lúdico Creativa, como son cursos de formación y talleres lúdico creativos en temas variados. 
ma de estar en la vida, de relacionarse con ella, en "espacios" y "ambientes" en los que se produce interacción, entretenimiento, disfrute, goce y felicidad, acompañados de la distensión que producen actividades simbólicas e imaginarias como el juego, la chanza, el sentido del humor, la escritura, el arte, el descanso, la estética, el baile, el amor, el afecto, las ensoñaciones, la palabrería, e inclusive todos aquellos actos cotidianos "como mirar vitrinas", "pararse en las esquinas", "sentarse en una banca", que también son lúdicos. (p.133).

Es una dimensión que trasciende toda la esencia del ser humano, su ser corporal, psíquico, social, cultural y espiritual. En esta misma línea, Araya (2016) expone que la lúdica se refiere a lo siguiente:

Una dimensión intrínseca y esencial a la naturaleza del ser humano, que se exterioriza por medio de una actitud hacia la vida, caracterizada por la alegría, el placer, el gozo, el disfrute, la esperanza, la creación, el asombro, la curiosidad, la imaginación, el ingenio, el buen humor, la espontaneidad, el caos y el orden, la tensión y la distensión: esta actitud encuentra su afirmación en múltiples tiempos, escenarios y expresiones culturales. (pp. 31-32)

Desde esta concepción no reduccionista, el ser humano es concebido como un ser integral y complejo, que está en constante interacción con el medio que le rodea, a la vez que es afectado positiva o negativamente por la dinámica que se genera en los ambientes en que se desenvuelve. En relación con la integralidad del ser humano, la profesora María Eugenia Dengo (1995) expone lo sucesivo:

El hombre es un ser unitario, una unidad compleja biopsicoespiritual y social. Como ser biológico que es, tiene cuerpo, sujeto a todas las condiciones de los seres vivos superiores; pero es mucho más que esto, ya que su espíritu supera la corporeidad para ser trascendencia. Al comunicarse con los otros hombres en relación social, en la que realiza su afectividad y en la mejor expresión de ésta, el amor, o al crear objetos culturales independientes a él mismo, trasciende igualmente su individualidad corporal. Como ser dotado de psique, está constituido internamente por una complejidad de intelecto, afectividad, voliciones, reacciones todas de su interioridad que se externalizan y se concretan en acciones y conductas. Como ser racional, lo que manifiesta la proyección de su espíritu (algunas escuelas filosóficas consideran que en esto radica su diferencia específica con respecto a otros seres vivos) es ser capaz de las más excelsas concepciones abstractas, de elevarse en la trascendencia religiosa, de establecer valores para orientar su conducta social e individual, de darle a su existencia una dimensión moral y de idear fundamentos teóricos para dirigir la vida práctica. (p.9).

Para alcanzar una mayor comprensión en relación con la lúdica, es vital referirnos a lo mencionado por la autora, ya que desde esta visión el ser humano tiene una constitución ontológica integral, por lo cual, Dengo (1995) considera que la educación debe procurar atender a su ser unitario, sin distanciar sus dimensiones existenciales, buscando su desarrollo integral a lo largo del tiempo.

Es así, que se considera que la escuela debe ser un ambiente en el cual se posibilite la actitud lúdica en las personas, ya que, como una dimensión del ser, puede ser exteriorizada de diversas maneras como la expresión corporal, oral, teatral, musical, entre otras, manifestaciones que favorecen el desarrollo integral de la persona y la búsqueda del significado de su vida.

Para Assman (2002), la escuela debe ser un lugar agradable que propicie la vinculación entre el conocimiento y el placer, tal como lo indica a continuación:

El ambiente pedagógico tiene que ser un lugar de fascinación e inventiva: no inhibir sino propiciar la dosis de ilusión común entusiasta requerida para que el proceso de aprender se produzca como mezcla de todos los sentidos. Transformación de los sentidos y significados, y potenciación de todos los sentidos con los que captamos corporalmente el mundo. Porque el aprendizaje es, antes que nada, un proceso corporal. Todo conocimiento tiene una inscripción corporal, y que venga acompañada de una sensación de placer no es, en modo alguno, un aspecto secundario. (p.28).

Además, Gutiérrez y Prieto (1993) consideran que en la escuela debe educarse para la significación, expresan que "Una educación con sentido educa protagonistas, seres para los cuales todas y cada una de las actividades, todos y cada uno de los conceptos, todos y cada uno de los proyectos, significan algo para su vida" (p.37).

Las perspectivas de Dengo, Assman y Gutiérrez y Prieto, reflejan la importancia de contar con ambientes pedagógicos lúdicos y creativos, que favorezcan el crecimiento integral del ser y posibiliten el encontrarle sentido a la vida.

En relación con el tema que se desarrolla, Jiménez (2005, p.133) considera que la lúdica "No es un estado, sino que

Salazar, M. y Montero C. Experiencias ludocreativas en niñas y niños de quinto y sexto grado de Costa Rica: una forma innovadora de sentir los espacios educativos. 
es toda la existencia humana, ya que, a través de los comportamientos lúdicos, el ser humano encuentra sentido a la vida, construyendo cultura y conocimiento". Además, Díaz (2008, p. 32) expresa que "Como función de equilibración emocional, la expresión lúdica constituye el mecanismo como el sujeto supera el conflicto emocional con la existencia y lo proyecta en vida".

En el trabajo lúdico creativo, desarrollado en ambas instituciones, se utiliza la metodología de taller:

(...) un dispositivo de trabajo con grupos, que es limitado en el tiempo y se realiza con determinados objetivos particulares, permitiendo la activación de un proceso pedagógico sustentado en la integración de teoría y práctica, el protagonismo de los participantes, el diálogo de saberes, y la producción colectiva de aprendizajes, operando una transformación en las personas participantes $\mathrm{y}$ en la situación de partida ${ }^{3}$. (Cano, 2012, p. 33).

En todo taller lúdico creativo las personas facilitadoras tienen la tarea de propiciar el rapport, entendido como un ambiente de empatía y realimentación que favorezca el desarrollo de la sesión, de manera que las personas participantes experimenten mayor apertura emocional y psicológica, así como la disposición para realizar las actividades planificadas desde una actitud lúdica.

El ambiente pedagógico lúdico creativo, se propicia desde el momento en que se define el nombre del taller, por lo que es primordial darle un nombre creativo que llame la atención de quienes participan en el mismo, ya que esto, provoca expectativa en las personas y orienta hacia el sentido de la actividad. Por esta razón, como se mencionó previamente, los talleres llevados a cabo se nombraron: “Soy creativ@y resuelvo mis conflictos!” y "Amistad y Esperanza: un paso al futuro". En nuestro caso, la lluvia de ideas fue la técnica utilizada para generar los nombres para los talleres, sin embargo, las técnicas a utilizar no deben limitarse, estas dependerán de las personas diseñen los talleres.

Se rescata también, que las actividades que se implementen a la hora del taller son el primordial medio para alcanzar los objetivos pedagógicos planteados. Entre los elementos principales que deben considerarse a la hora de plantear las actividades, están las siguientes: a) propiciar el disfrute y goce entre los participantes, b) tener intencionalidad pedagógica, c) considerar características de las personas como: edad, zona geográfica de procedencia o lugar donde se desarrolla la actividad; entre otros que se consideren necesarios para favorecer la mayor participación en las actividades.

Por este motivo, los talleres iniciaron con un encuadre, que incluye una bienvenida amistosa para promover la emoción, la alegría, el entusiasmo y la convivencia, sin obviar el respeto a las normas que se establecieron previamente, las cuales se mencionan a continuación:

1. Respetar las indicaciones de las personas facilitadoras.

2. Apagar los celulares u otros aparatos electrónicos.

3. Levantar la mano para participar.

4. Respetar el espacio verbal de los demás compañeros.

5. No salir del salón cuando se está en la sesión, a menos que sean emergencias.

Las normas, aunque fueron previamente establecidas por las personas facilitadoras, las mismas fueron propuestas de forma oral a los participantes y al momento del taller se les preguntó si desean proponer algunas otras, además, se motivó al compromiso grupal por respetarlas.

Seguidamente se hace una presentación personal creativa para llamar a cada persona por su nombre, así como el desarrollo de algunas actividades rompehielos, que cumplan su función de generar un ambiente y actitud de distensión, asombro e imaginación (Araya, 2016).

Tomando en cuenta que los talleres propician un dinamismo entre teoría y práctica que facilita el aprendizaje significativo, es importante definir este concepto.

El aprendizaje significativo, es según Molina (2004, p.135), aquél que se da cuando el estudiante "como constructor de su propio conocimiento, relaciona los contenidos por aprender y les da un sentido, a partir de los conocimientos que ya posee.", además, este aprendizaje supone "modificar los esquemas conceptuales que el alumno

3 La conceptualización de Cano de la noción de dispositivo se relaciona con el desglose conceptual realizado por el filósofo Giorgo Agambem acerca de una de las piezas fundamentales en el sistema de pensamiento del filósofo Michel Foucault. Según Fernández (2007, p.115) en Cano (2012, p.36) "El dispositivo es pensado como máquina que dispone a..., que crea condiciones de posibilidad, que provoca o pone en visibilidad y eventualmente en enunciabilidad latencias grupales, institucionales y/o comunitarias".

Salazar, M. y Montero C. Experiencias ludocreativas en niñas y niños de quinto y sexto grado de Costa Rica: una forma innovadora de sentir los espacios educativos. 
posee; esto implica partir de la realidad del alumno e impulsarlo a desarrollar su potencial de aprendizaje".

También, Rogers (1978) citado por Hernández (2006) indica que el aprendizaje llega a ser significativo cuando "involucra a la persona como totalidad (procesos afectivos y cognitivos) y se desarrolla de forma experiencial (que se entreteja con la personalidad del alumno)" (p.111).

El dinamismo del que se habló anteriormente se genera cuando los elementos teóricos que fundamentan el taller se relacionan con el desarrollo de las actividades que se llevan a la práctica. En el caso de los talleres: "iSoy creativ@y resuelvo mis conflictos!” y “Amistad y Esperanza: un paso al futuro", se trabajó en pequeños subgrupos, las formas diferenciadas en las que los participantes se comunican y perciben, según las características de su personalidad, historias de vida o estados de ánimo.

En relación con las actividades planteadas, es importante rescatar que, si bien ambos talleres compartieron un objetivo general común, la planificación correspondió a las características de las personas participantes, por lo cual, las actividades son diferentes para ambos grupos.

En el caso del taller "Amistad y Esperanza: un paso al futuro", que se realizó con estudiantes de sexto grado de la escuela Bernardo Soto es un espacio al aire libre y con ranchos en El Coyol de Alajuela, se llevaron a cabo las actividades mostradas a continuación:

Tabla 3. Resumen de Actividades del taller Amistad y Esperanza: un paso al futuro.

Tabla resumen de actividades del taller Amistad y Esperanza: un paso al futuro

\begin{tabular}{|c|c|c|c|}
\hline $\begin{array}{c}\text { OBJETIVO } \\
\text { ESPECÍFICO }\end{array}$ & ACTIVIDADES & MATERIALES & TIEMPO \\
\hline $\begin{array}{l}\text { Que los estudiantes se sien- } \\
\text { tan cómodos y motivados } \\
\text { para participar del taller des- } \\
\text { de una actitud lúdica. }\end{array}$ & $\begin{array}{l}\text { Petividad de Presentación perso- } \\
\text { nal } \\
\text { Personalización creativa de una hoja } \\
\text { que conformó un mural, en donde } \\
\text { los participantes se escribieron men- } \\
\text { sajes positivos. Además, cada parti- } \\
\text { cipante presentó su hoja a los demás. } \\
\text { Rompehielos } \\
\text { Hula Hula, Números y Manicomio } \\
\text { fueron los tres rompehielos reali- } \\
\text { zados, consisten en actividades de } \\
\text { coordinación grupal, uso de la lógica } \\
\text { y movimiento. }\end{array}$ & $\begin{array}{l}\text { Música. } \\
\text { Parlante. } \\
\text { Micrófono. } \\
\text { Cable de audio. } \\
\text { Proyector. } \\
\text { Extensiones. } \\
\text { Explicación oral de normas. } \\
\text { Lista de asistencia. } \\
\text { Lapiceros. } \\
\text { Hojas de colores. } \\
\text { Marcadores. } \\
\text { Calcomanías. } \\
\text { Cinta. } \\
\end{array}$ & $20 \mathrm{~min}$ \\
\hline
\end{tabular}




\begin{tabular}{|c|c|c|c|}
\hline $\begin{array}{l}\text { Que los estudiantes expresen } \\
\text { emociones y sentimientos a } \\
\text { sus compañeros como una } \\
\text { habilidad social que favorece } \\
\text { la resolución pacífica de con- } \\
\text { flictos. }\end{array}$ & $\begin{array}{l}\text { Tiempo de esparcimiento y me- } \\
\text { rienda } \\
\text { En este momento los estudiantes pu- } \\
\text { dieron tomarse fotografías en el pho- } \\
\text { tobooth y escribieron mensajes posi- } \\
\text { tivos en las hojas de sus compañeros } \\
\text { que se encontraban en el mural. }\end{array}$ & $\begin{array}{l}\text { Marco de photobooth } \\
\text { Marcadores } \\
\text { Música }\end{array}$ & $30 \mathrm{~min}$ \\
\hline $\begin{array}{l}\text { Que los estudiantes pongan } \\
\text { en práctica los pasos para } \\
\text { resolver conflictos de forma } \\
\text { pacífica. }\end{array}$ & $\begin{array}{l}\text { Rally } \\
\text { Se realizó un rally de ocho estacio- } \\
\text { nes, donde los estudiantes tuvieron } \\
\text { que resolver los diversos desafíos } \\
\text { presentados, para ello se dividieron } \\
\text { en equipos. } \\
\text { Las estaciones se denominaron: } \\
\text { Esponjas } \\
\text { Balde con vasos } \\
\text { Las } 3 \text { Piñas } \\
\text { Balde con soperita y moneda } \\
\text { Portabolas } \\
\text { Cápsula } \\
\text { Suiza } \\
\text { Alfombra } \\
\text { Al finalizar, se hizo una plenaria. }\end{array}$ & $\begin{array}{l}\text { Rompecabezas } \\
\text { Limones } \\
\text { Conos } \\
\text { Esponja grande } \\
\text { Baldes } \\
\text { Vasos de plástico } \\
3 \text { piñas } \\
\text { Soperita } \\
\text { Moneda } \\
\text { Portabolas } \\
\text { Bolos } \\
\text { Telas negras } \\
\text { Mecate largo } \\
\text { Plástico negro grande } \\
\end{array}$ & $1: 30 \mathrm{~min}$ \\
\hline \multicolumn{4}{|c|}{ Tiempo de almuerzo y compartir } \\
\hline $\begin{array}{l}\text { Que los estudiantes se refuer- } \\
\text { cen positivamente y reflexio- } \\
\text { nen sobre la importancia del } \\
\text { amor propio en las relaciones } \\
\text { consigo mismo y los demás, } \\
\text { como una habilidad social } \\
\text { que favorece la resolución } \\
\text { pacífica de conflictos. }\end{array}$ & $\begin{array}{l}\text { Círculos de virtudes } \\
\text { Los estudiantes se sentaron en dos } \\
\text { círculos (uno dentro del otro) que- } \\
\text { dando frente a frente, el círculo in- } \\
\text { terior se quedó fijo y el exterior se } \\
\text { movilizó a la derecha. Cada compa- } \\
\text { ñero dijo una virtud o fortaleza real } \\
\text { al otro y viceversa. Todos se rotaron } \\
\text { hasta que todos se expresaron sus } \\
\text { fortalezas, mientras uno hablaba el } \\
\text { otro escuchaba con atención. } \\
\text { Al finalizar, se hizo una plenaria. } \\
\text { Posteriormente, se invitó al grupo a } \\
\text { que quienes deseaban se brindaran } \\
\text { un respetuoso abrazo y a pedir per- } \\
\text { dón a algún compañero. }\end{array}$ & $\begin{array}{l}\text { Sillas. } \\
\text { Guía de preguntas. } \\
\text { Parlantes } \\
\text { Música de fondo. } \\
\text { Canción "Qué difícil se me } \\
\text { hace" de Nicho Hinojosa. }\end{array}$ & $30 \mathrm{~min}$ \\
\hline
\end{tabular}




\begin{tabular}{|c|c|c|c|}
\hline $\begin{array}{l}\text { Que los estudiantes en con- } \\
\text { junto planteen metas, expec- } \\
\text { tativas y esperanzas para el } \\
\text { futuro que desean para toda } \\
\text { su generación. }\end{array}$ & $\begin{array}{l}\text { Manta de la esperanza } \\
\text { Se les proporcionó a los participan- } \\
\text { tes una tela grande blanca, pinturas } \\
\text { de colores, pinceles y marcadores. } \\
\text { Se les invitó a plasmar en la tela sus } \\
\text { metas, sueños, expectativas y espe- } \\
\text { ranzas para el futuro cercano, asi- } \\
\text { mismo pudieron señalar elementos } \\
\text { que deseaban dejar atrás. } \\
\text { Reflexión final a cargo de las faci- } \\
\text { litadoras. }\end{array}$ & $\begin{array}{l}\text { Tela grande blanca } \\
\text { Temperas } \\
\text { Marcadores } \\
\text { Pinceles }\end{array}$ & $15 \mathrm{~min}$ \\
\hline $\begin{array}{l}\text { Que los estudiantes evalúen } \\
\text { el taller y los aprendizajes al- } \\
\text { canzados. }\end{array}$ & $\begin{array}{l}\text { Evaluación del taller. } \\
\text { Cierre de taller y despedida. }\end{array}$ & $\begin{array}{l}\text { Hojas de evaluación. } \\
\text { Lapiceros. }\end{array}$ & $5 \mathrm{~min}$ \\
\hline
\end{tabular}

Fuente: elaboración propia, 2019.

Con respecto al taller “iSoy creativ@y resuelvo mis conflictos!” que se realizó con estudiantes de quinto grado de la escuela Mixta San Juan en San José, se llevaron a cabo las siguientes actividades:

Tabla 4. Resumen de Actividades del taller ¡Soy creativ@ y resuelvo mis conflictos!

Tabla resumen de actividades del taller ¡Soy creativ@y resuelvo mis conflictos!

\begin{tabular}{|c|c|c|c|}
\hline $\begin{array}{c}\text { OBJETIVO } \\
\text { ESPECÍFICO }\end{array}$ & ACTIVIDADES & MATERIALES & TIEMPO \\
\hline $\begin{array}{l}\text { Que los estudiantes se sien- } \\
\text { tan cómodos y motivados } \\
\text { para participar del taller } \\
\text { desde una actitud lúdica. } \\
\text { Que los estudiantes se brin- } \\
\text { den mutuamente refuerzo } \\
\text { positivo que les permita } \\
\text { reconocer las fortalezas } \\
\text { personales y del otro, como } \\
\text { una habilidad que favorece } \\
\text { la resolución pacífica del } \\
\text { conflicto. }\end{array}$ & $\begin{array}{l}\text { Actividad de Presentación personal } \\
\text { Personalización creativa de una hoja } \\
\text { que conformó un mural, en donde los } \\
\text { participantes se escribieron mensajes } \\
\text { positivos. Además, cada participante } \\
\text { presentó su hoja a los demás. } \\
\text { Rompehielos } \\
\text { Hula Hula, Números y Manicomio } \\
\text { fueron los tres rompehielos realizados, } \\
\text { consisten en actividades de coordina- } \\
\text { ción grupal, uso de la lógica y movi- } \\
\text { miento. }\end{array}$ & $\begin{array}{l}\text { Música. } \\
\text { Parlante. } \\
\text { Micrófono. } \\
\text { Cable de audio. } \\
\text { Proyector. } \\
\text { Extensiones. } \\
\text { Explicación oral de normas. } \\
\text { Lista de asistencia. } \\
\text { Lapiceros. } \\
\text { Hojas de colores. } \\
\text { Marcadores. } \\
\text { Calcomanías. } \\
\text { Cinta. } \\
\\
\text { 2 Hula Hula } \\
\text { Sillas para cada participante } \\
\text { Cinta } \\
\text { Marcadores }\end{array}$ & $20 \mathrm{~min}$ \\
\hline
\end{tabular}


Tiempo de almuerzo y compartir

$40 \mathrm{~min}$

Que los estudiantes pongan en práctica la tolerancia y respeto por las diferencias individuales entre los compañeros, como elementos que favorecen la resolución pacífica de conflictos.

\section{¿Será que podemos?}

Es una actividad en grupos. Las personas debieron armar un carro original y diferente, aprovechando los materiales brindados (pajillas, papel ceda, cajas de cartón, cinta adhesiva); pero se les puso un desafío al limitarles la comunicación grupal: a uno se le vendaron los ojos, a otro la boca y otros las manos, de manera que no podían hablar, ver o utilizar las manos para construir el vehículo, según correspondió. Una vez construido, lo presentaron a sus demás compañeros, a manera de venta.

Al finalizar, se hizo una plenaria.

Que los estudiantes trabajen en equipo y alcancen una meta conjunta a pesar de las diferencias personales.

\section{La Torre del compañerismo}

Es una herramienta que favorece la construcción de equipo, posibilita la comunicación efectiva, cooperación y la escucha activa.

Las facilitadoras colocaron 8 bloques de madera sobre el piso, cada bloque contiene una palabra de la frase ¡Juntos somos más fuertes!, por lo que se le pidió al grupo que arme la frase.

Posteriormente, cada miembro del equipo tomó una cuerda que estaba conectada a una grúa. La grúa se usó para construir una torre colocando los bloques uno encima del otro en el orden de la frase brindada. Los participantes no pudieron tocar los bloques con sus manos, ni con ninguna parte del cuerpo.

Al finalizar, se hizo una plenaria.

Evaluación del taller.

Cierre de taller.
Vendas.

Cajas de cartón. Pajillas.

Cinta adhesiva. Papeles.

Marcadores.

Tijeras.

Gomas.
Que los estudiantes evalúen el taller y los aprendizajes alcanzados.

Fuente: elaboración propia, 2019. 
En este último taller, en un inicio se planificó realizar la actividad círculo de virtudes, sin embargo, la dinámica grupal llevó a que las actividades ¿Será que podemos? y la Torre del compañerismo, requirieran más tiempo para su desarrollo. Por lo que, en ese momento se tomó la decisión de no llevarla a cabo.

Como se presenta en las tablas 3 y 4, en algunas de las actividades para la resolución pacífica de conflictos interpersonales, las personas participantes se enfrentaron a una situación "hipotética" que requería la resolución de un problema.

Molina (2004, p.105), expone que "las actividades de construcción deben poner al alumno ante un reto por resolver, ya sea un hacer, un retomar lo que se conoce sobre el tema, una situación crítica o problemática", además, indica que "Una vez asumido el reto por parte de los alumnos, viene la búsqueda de soluciones al problema, la explicación de la inquietud, o el momento de analizar y deducir lo que ocurrió durante el experimento o la prueba realizada.".

Al enfrentarse a las situaciones donde debían resolver una problemática, las niñas y los niños que participaron, generaron un desafío en cuanto a su forma de convivir, de comunicarse, de observarse y de comprenderse, puesto que mediante el grupo se posibilita el intercambio de respuestas ante una situación, así como el cuestionamiento y validación de las diversas formas de reaccionar y expresarse ante un mismo evento. En relación con el aporte de la socialización en las situaciones de aprendizaje Molina (2004) expone que "las actividades grupales constituyen un medio esencial para garantizar el aprendizaje socializado, compartido y solidario" (p.106).

Uno de los elementos más relevantes de los talleres lúdico creativos, tiene que ver con la relación dialógica que se da entre las personas facilitadoras y las participantes tanto individual como grupalmente, así como de la pedagogía de la pregunta ${ }^{4}$.

Para Dengo (1995) el ser humano es un ser dialógico que requiere comunicarse, expresarse, manifestarse con las otras personas, además de recibir la realimentación de parte de ellas. Esta autora considera que "el diálogo es la base de la solución de los conflictos humanos, es la posibilidad de entendimiento y comprensión" (p.12), además expone que el proceso de enseñanza y aprendizaje es un diálogo entre educador y estudiante.

A lo largo de los talleres, se propició el diálogo y la expresión de ideas, emociones y sentimientos de los participantes desde una actitud lúdica, además, se planificó espacios de plenaria al concluir las actividades.

Los escolares comentaron voluntariamente qué sintieron, pensaron y experimentaron, y las personas facilitadoras se dieron a la tarea de realizar un análisis de la información emitida por los estudiantes, de los elementos que conforman la dinámica grupal para plantear nuevas preguntas generadoras centradas en la experiencia vivida. Finalmente, se realizó una realimentación acerca de las experiencias vividas, entre las que se señalan las expresiones verbales y no verbales que subyacen como parte de la experiencia, a la vez que se brindaron elementos teóricos que fundamentaron el tema que se está tratando y se verifica al logro de los objetivos del taller.

La formulación de las preguntas generadoras para un taller debe permitir que las personas participantes puedan expresarse libremente sin sentirse intimidadas, sino que promuevan la reflexión acerca del tema. Por este motivo, se plantearon preguntas abiertas que motiven a una conversación sana y sin prejuicios.

Las personas facilitadoras deben promover la participación de la mayor cantidad de participantes, son flexibles, aprovechando las respuestas para lograr una efectiva y eficiente dinámica.

A la vez, la relación comprensiva y espontánea entre las personas que diseñan, ejecutan y evalúan los talleres es vital para enriquecer cada etapa de la experiencia lúdico creativa.

Las actividades planificadas, al conllevar una intencionalidad pedagógica de parte de los facilitadores, no solo favorecen el logro de los objetivos planteados, sino que se toman en consideración otros elementos como la edad

$4 \quad$ La pedagogía de la pregunta, según Gutiérrez y Prieto (1993), “constituye una de las formas pedagógicas más importantes de todo aprendizaje" (p.73). Entre los aspectos que comprende están: a) todo contenido puede convertirse en una pregunta, b) debe definirse el momento preciso para formular una pregunta, c) identificar si se requiere una pregunta abierta o cerrada según el contexto, d) hay preguntas sin respuesta, pero eso no significa que pierdan su carácter pedagógico, e) las preguntas conllevan temporalidad y, f) las preguntas pueden hacer referencia a los contenidos, pero también a los ángulos de mira. 
de las niñas y los niños, la duración del taller, el contexto escolar y el sociocultural de la población.

Como lo menciona CONARE (2009b) citado por Pérez-Yglesias (2010), el conocimiento y el aprendizaje se alcanzan mediante "talleres innovadores en donde se conjuguen las diversas expresiones: música, arte, danza, juego, psicomotricidad, teatro, literatura, creatividad, humor y otras, con el fin de favorecer el desarrollo de los diferentes talentos personales como profesionales" (p. 69). Por lo tanto, los talleres “iSoy creativ@y resuelvo mis conflictos!" y "Amistad y Esperanza: un paso al futuro", vivenciado por la población escolar mencionada, llevó a la práctica con gran éxito, algunas de las expresiones que cita Pérez-Yglesias, las cuales fueron mencionadas anteriormente.

Cabe señalar que, como parte de la organización, también se contemplaron espacios de alimentación y receso, considerados vitales para la experiencia lúdica.

Por último, se valida la experiencia vivida por cada persona en el taller, por medio de un instrumento llamado "Valoración del taller lúdico creativo". El cual permite la perspectiva de los participantes en cuanto a los aprendizajes alcanzados y la metodología aplicada.

Las respuestas ofrecidas por la población estudiantil por medio de las evaluaciones ${ }^{5}$ sirvieron de marco para la reflexión y sistematización de los aprendizajes desarrollados en este artículo.

Precisamente las respuestas que ofrecen los estudiantes son las que ayudan a sistematizar la experiencia, pues se clasifican en categorías y hay suficiente materia para la sistematización.

\section{Reflexionando con los aprendizajes \\ "Amé que nos trataran bien" \\ Estudiante, 12 años}

El taller es ideal para propiciar una actitud ludocreativa en las niñas y niños, ya que genera ambientes que promueven la creatividad, la expresión de emociones, sentimientos e ideas y, al compartir, no solo con la persona facilitadora o profesora (díada tradicional sino con sus coetáneos experiencias ricas en sensaciones, esas acciones se pueden convertir en 1 aprendizajes para la construcción colectiva de nuevos saberes.

Así, del total de 59 niñas y niños que participaron y evaluaron el taller, un $88 \%$ manifiestan que la organización y planificación general de las actividades fueron divertidas y muy buenas. Una participante de 11 años afirma: "Me gustó mucho estuvo divertido más la guerra de pintura ${ }^{6 "}$. También, en la misma línea, un 5\% expresó que los materiales fueron los adecuados para la ejecución del taller; un participante de 10 años indicó que "son materiales buenos y que no contaminan".

Además, el $7 \%$ se refirió específicamente a la relación que se generó con las facilitadoras del taller, al respecto una participante de 12 años indica "Me gustó mucho la experiencia y las actividades, pienso que las muchachas nos dieron muy buen trato".

Vivir un acto pedagógico desde la dimensión lúdica es fundamental, en relación con esto Araya (2016) indica lo siguiente:

Cuando los docentes son lúdicos, son conscientes de sí mismos, de su valor como persona, de sus capacidades, de su inteligencia, de su voluntad, de su unicidad en y con el universo; es consciente de cada uno de sus respiros, de lo fascinante de relacionarse cada día a partir de sus cinco sentidos. (p. 154).

Esta actitud ante la vida favorece que los "ludo maestros" desarrollen de forma consciente actos pedagógicos lúdicos, a la vez, que posibilita que se generen vínculos tier-

5 El instrumento utilizado es una evaluación estándar escrita aprobada para todas las actividades de la Subcomisión, cuenta con preguntas respecto a la edad y al género, una introducción y cuatro preguntas: ¿Que piensa respecto al taller recibido (materiales, expositores, organización, etc.)? / ¿Qué aprendizajes le generó esta actividad? / ¿Qué sentimientos tuvo durante el taller? / Otros comentarios.

6 A partir de este momento empiezan a aparecer frases de las personas participantes de los talleres que, incluso, fueron escritas con errores de ortografía o de redacción, pero se decidió respetar la forma y citarlas tal cual aparecen en los instrumentos de evaluación. Como afirma Beluche (2019), "A veces en forma textual debidamente citadas, otras veces mediante la escritura del autor, intentando con respeto transmitir e interpretar de la manera más fiel lo escuchado y conversado. La intención es hacer que sus voces se oigan y que queden escritas de forma indeleble en este documento" (p.109).

Salazar, M. y Montero C. Experiencias ludocreativas en niñas y niños de quinto y sexto grado de Costa Rica: una forma innovadora de sentir los espacios educativos. 
$\operatorname{nos}^{7}$ con las personas participantes de las experiencias.

Como podemos ver, los participantes destacan la organización y planificación de los talleres, elemento vital para desarrollar la metodología lúdico creativa. En esta línea, se rescata que, para generar ambientes lúdicos, es necesario el diseño intencionado del taller para el logro de objetivos pedagógicos que fomenten las características de la actitud lúdica (Araya, 2016) como la creación, la espontaneidad, el asombro, curiosidad e ingenio.

En relación con lo anterior, la misma autora señala que para generar un ambiente lúdico, es necesario prepararse, como lo indica seguidamente:

la preparación consciente intencional anticipada y sistemática de un ambiente pedagógico, constituido por un espacio social (interacciones sociales) y un espacio físico (ambiente natural, la infraestructura y los materiales), que inviten a vivir la vida y la cotidianidad pedagógica desde la dimensión lúdica (p. 147).

Sin embargo, aunque exista una coherente e innovadora planificación, las personas facilitadoras también deben poseer una excelente formación académica y pedagógica, además de sentir orgullo y satisfacción por lo que hacen, sin obviar que sus cualidades personales les permitan ser flexibles a la hora de ejecutar el taller, de modo que sean capaces de crear sus propias prácticas pedagógicas a partir de una fundamentación teórica clara, para atender las necesidades de sus estudiantes en beneficio de su desarrollo integral..

Como afirma Paredes (2003) "la ludocreatividad corresponde a un elemento metodológico ideal para dotar a los niños de una formación integral." (p.12). Respecto a este tema, el 88,13\% de las personas participantes manifiestan que uno de los aprendizajes que se construyeron en las sesiones fue la importancia del compañerismo y la convivencia pacífica, así lo manifiesta una niña de 12 años, quien afirma haber aprendido "A querer más a mis compañeros, y me di cuenta de que tenía más cosas buenas de las que pensaba." y un niño de 12 años indicó que aprendió "A compartir mucho y estar con mis amigos".
Entendemos que, "Las experiencias son individuales y colectivas a la vez, las vivimos y nos hacen vivir; somos seres humanos en cuanto vivimos cotidiana y socialmente las experiencias de las que somos sujetos y objetos al mismo tiempo" (Jara, 2012, p.69). Acerca de la cita anterior, se rescata que la experiencia lúdica grupal, favoreció la construcción de aprendizajes significativos colectivos, que posibilitaron el intercambio de saberes y vivencias que realimentaron la vida personal.

Otro de los aprendizajes que se vivenciaron fue la promoción de la convivencia pacífica y la resolución de conflictos, ya que, durante la interacción, las personas tenemos la posibilidad de ir más allá de nosotras mismas, encontrar sentido y autotrascender. Como lo expone Noblejas (2018):

Así, el buscar la felicidad no puede ser otra cosa que expresar nuestra capacidad de donación, de salir de nosotros mismos, sin dejar de ser nosotros mismos. Por el contrario, cuanto más se esfuerza el hombre en perseguir la felicidad centrándose en sí mismo, en la propia autosatisfacción de necesidades, más se incapacita a sí mismo para lograrla, ya que con esta actitud está negando su propia constitución, la autotrascendencia, y su expresión fundamental, la voluntad de sentido. (p. 73).

Acorde con el texto anterior, es en el encuentro con el otro, donde se nos brinda la oportunidad de traspasar nuestra propia existencia e intimar con la de las demás personas, es decir, de autotrascender, generando un efecto positivo en su ambiente y/o viceversa. Justamente, Frankl (1995) indicó lo siguiente:

“...sólo en la medida en que el hombre va más allá de sí mismo, se realiza también a sí mismo: en el servicio a algo o en el amor hacia otra persona. Cuanto más se entrega a su tarea, cuanto más se entrega a su pareja, es más persona, es más él mismo" (p. 79).

Acerca de la convivencia, Noblejas (2018) a continuación expone:

Todo ser humano, como ser-en-el-mundo, en cuya constitución radica el encuentro con el otro (no puede vivir solo, necesita convivir y humanizarse en la

7 La propuesta pedagógica de la ternura, según Araya (2016), “indica que todo acto pedagógico tenga presente la ternura, como esta maravillosa posibilidad de expresión de la afectividad, que permite la sensibilidad ante el otro y la vida que lo rodea, lo cual es básico en las interacciones sociales características del acto pedagógico; que el docente sea consciente de la ternura de todo ser humano; y que exista, en todo ambiente pedagógico, es posibilidad de relacionarse con los otros al considerar esta forma de expresión, la cual acerca y une desde esa capacidad para sensibilizarse ante los otros seres humanos y seres vivos en general, con quienes se construye y se comparte el conocimiento.” (p.254).

Salazar, M. y Montero C. Experiencias ludocreativas en niñas y niños de quinto y sexto grado de Costa Rica: una forma innovadora de sentir los espacios educativos. 
interacción y salida de sí mismo), siente la necesidad de encontrar sentido en su proyecto de vida, en la familia y en la sociedad, de escribir su propia historia vital y expresar lo propio, su compromiso existencial, en la participación, el diálogo y la reflexión. (p. 72)

Se reitera que la dinámica grupal aplicada nos ha permitido comprender que todas las personas somos distintas, tanto en cualidades como en aspectos por mejorar, tal y como lo expresa un niño de 11 años, quien aprendió "que todos tienen cualidades distintas".

Consideramos fundamental promover el desarrollo de habilidades (qué tipo de habilidades) para fomentar el compañerismo y la resolución pacífica de conflictos que permitan convivir desde el respeto a las diferencias, como lo afirma Coeetze y Kurtz (2015):

Me parece que la facultad de ver a los otros como seres complejos, capaces de cosas buenas, malas y de todos los grises intermedios, más la posibilidad de tolerar la frustración y la ambivalencia son esenciales para participar constructivamente de la vida grupal. (p. 178)

En relación con las emociones experimentadas en los talleres, Paredes (2003) afirma que "La enseñanza debe caminar hacia una participación más activa por parte del niño en el proceso educativo. Hay que fomentar la actividad lúdica como cualquier otra actividad que conlleve el compromiso, la curiosidad, la creatividad, la inteligencia emocional. Tan importante como adquirir es sentir los conocimientos." (p.12).

Si se toma en cuenta la cita anterior, la población participante del taller, señalan que en un 50,84 \% experimentaron emociones relacionadas con la felicidad, alegría y amor; por otra parte, también se destaca que un 38,98\% sintieron emociones variadas, e incluso ambivalentes: "Lloré, reí y de todo un poco". (Mujer, 12 años)"; "Me divertí mucho y me puse muy sentimental con mis amigos." (Hombre, 14 años); "Emoción, felicidad, tristeza, adrenalina, frustración, etc." (Hombre, 12 años).

Además, el 8,47 \% de las personas participantes indicaron únicamente que lloraron o sintieron tristeza y el 1,69 $\%$ indicó no sentir ninguna emoción al respecto.

La dimensión lúdica es inherente al ser humano y el vivir emociones variadas es parte de nuestra naturaleza, por lo tanto, que los niños y las niñas sintieran alegría, tristeza, frustración, entre otras emociones, en un corto tiempo, permite visualizar que las actividades de corte lúdico creativo potenciadoras del autoconocimiento, la identificación de emociones y la capacidad de coherencia emocional según la situación vivida.

Para analizar las experiencias ludocreativas de las personas participantes de los talleres, fueron vitales las evaluaciones escritas, en ellas podemos construir saberes dialogando con las palabras de las personas participantes, así entendemos que "la palabra no solo vehicula sonidos, sino sobre todo sentires, emociones, afectos, sensibilidades, vibraciones del espíritu que van construyendo un sentido sobre la existencia, sobre la realidad y la vida (...)" (Guerrero, 2010, pp. 288-289).

Un taller debe convertirse en una experiencia significativa para la construcción de saberes que perduren e impacten de manera positiva en toda población participante. La construcción de conocimientos produce transformaciones en las áreas cognitiva, socio afectiva y psicomotora. Además, se considera fundamental para que la población escolar pueda analizarlos, discutirlos, reelaborarlos y expresarlos mediante recursos musicales, plásticos y poéticos. Por lo tanto, se considera que las experiencias pedagógicas que se llevaron a cabo en los centros educativos de la escuela Mixta San Juan de Puriscal en San José y en la escuela Bernardo Soto Alfaro de Alajuela, resultaron exitosas, ya que el $61 \%$ de los comentarios, plantea aspectos positivos y se enfocan en lo divertido de la experiencia y en el buen trabajo de las facilitadoras.

Para muestra queremos concluir con un comentario y dibujos escritos en una de las evaluaciones de una de las participantes con respecto al taller: "es una experiencia que nunca voy a olvidar. Me encanto la actividad, estuvo muy vonita. $\vee$ ツ” (Mujer, 12 años).

\section{Conclusiones}

"La investigación es una oportunidad para resignificar nuestro saber y gestar espacios educativos pertinentes para el proyecto social comunitario que necesitamos"

Giovanni Beluche

El trabajo colaborativo entre las carreras de Orientación, Trabajo Social y Antropología, en el diseño, ejecución y evaluación de los talleres fue de suma importancia para lograr experiencias pedagógicas lúdico creativas. Siendo el trabajo conjunto una característica que se destaca en los proyectos de Extensión y Acción Social. 
La actitud lúdica de las personas facilitadoras es vital para la creación de ambientes pedagógicos lúdico creativos, ya que en su esencia el docente o quien facilite una actividad, debe experimentar en sí mismo el disfrute, el goce, el placer, la tensión y distensión, la imaginación, la esperanza, entre otras manifestaciones de la dimensión lúdica, lo cual a su vez genera un impacto en las vidas de las personas participantes.

La estructura metodológica de un taller lúdico creativo es tan importante como la intencionalidad pedagógica, ya que esto permite trascender y desmitificar la ludocreatividad exclusivamente como juego o diversión, lográndose experiencias enriquecedoras en su forma y contenido.

La ludocreatividad en una forma de innovar el espacio pedagógico, ya que, permite una vivencia enraizada en el presente y en el espacio geográfico. Se trata de una actividad que permite una vivencia de aprendizaje en el aquí y el ahora.

La ludocreatividad innova el espacio pedagógico, ya que los estudiantes al desarrollar la actitud lúdica traspasa los límites del ambiente de aprendizaje formal, el que se da en la escuela con las actividades cotidianas, permitiendo que esta actitud permee tanto los espacios formales como los cotidianos.

La ludocreatividad facilitó el reconocimiento de sentires de los participantes que, incluso eran emociones y sentimientos ambivalentes, como una forma del autoconocimiento personal. Este reconocimiento se facilita principalmente en la interacción y dinámica grupal con los compañeros, permitiendo la validación de las propias emociones como las de los demás.

Se evidencia que el reforzamiento positivo beneficia grandemente las relaciones de amistad y compañerismo, ya que, previene el conflicto al proveer a los niños y las niñas de estímulos de interacción positivos.

La experiencia ludocreativa favoreció que las personas participantes se encontraran con otros estudiantes y construyeran, de manera colectiva nuevos aprendizajes. Lo esencial fue la reflexión acerca de la importancia de usar las habilidades sociales en la resolución del conflicto en la relación con las demás personas, y cómo usando las habilidades de escucha activa, empatía, reforzamiento positivo, expresión de emociones y sentimientos, y resolución pacífica de conflictos, se evitan y solucionan problemas en un grupo.
A pesar de las diferencias entre las personas participantes, se evidenció cohesión grupal y actitud empática en la solución de los problemas planteados.

En la vinculación con otras personas, es posible hallar rutas que guíen hacia el encuentro del sentido de la vida.

El artículo de sistematización permitió por medio de la escritura profundizar la reflexión de los vínculos Universidad-Sociedad.

\section{Referencias}

Araya Góchez, A.P. (2016). La dimensión lúdica del ser humano en el aprendizaje. San José, Costa Rica: Editorial Universidad Estatal a Distancia.

Assman, Hugo. (2002). Placer y ternura en la educación. Hacia una sociedad aprendiente. Madrid: NARCEA S. A. DE EDICIONES

Beluche, G. (2019). Educación para el Buen Vivir. Saberes y sentires del pueblo Ngäbe. Costa Rica: Editorial Universidad Técnica Nacional

Cano, A. (2012). La metodología de taller en los procesos de educación popular. Revista Latinoamericana De Metodología De Las Ciencias Sociales (Relmecs), (2), 22-52. Recuperado de https://www. relmecs.fahce.unlp.edu.ar/

Consejo Nacional de Rectores. Comisión de Vicerrectores de Extensión y Acción Social. Planes de trabajo 2017 del área de extensión y acción social [Recurso electrónico]. San José, Costa Rica: CONARE-OPES

Díaz M, Hector Angel. (2008). Hermenéutica de la lúdica y pedagogía de la modificabilidad simbólica. Bogotá: Cooperativa Editorial Magisterio. ISBN 9582009713, 9789582009717

Dengo, María Eugenia. (1995). Educación Costarricense. Costa Rica: Editorial Universidad Estatal a Distancia.

Fantova, F. (2003). La sistematización como herramienta de gestión. Sistematización de experiencias de desarrollo humano del Instituto Pedro Arrupe de Derechos Humanos (Universidad de Deusto). Recuperado de https://www.alboan.org

Guerrero Arias, Patricio (2010). Corazonar. Una antropología comprometida con la vida. Quito: Ediciones ABYA Yala: Universidad Politécnica Salesiana. 
Gutiérrez, F. y Prieto, D. (1993). La Mediación Pedagógica: apuntes para una educación a distancia alternativa. Guatemala: Instituto de Investigaciones y Mejoramiento Educativo IIME.

Hernández, G. (2006). Paradigmas en psicología de la Educación. México, D.F.: Editorial Paidós

Jara Holliday, Oscar. (2012). La sistematización de Experiencias, Práctica y Teoría para Otros mundos posibles. San José, Costa Rica. Centro de Estudios y Publicaciones Alforja.

Jiménez Vélez, Carlos Alberto. (2005). La inteligencia lúdica: juegos y neuropedagogía en tiempos de transformación. Bogotá: Cooperativa Editorial Magisterio. ISBN 9582008288, 9789582008284

Molina Bogantes, Zaida. (2004). Planeamiento didáctico: fundamentos, principios y procedimientos para el desarrollo. San José, Costa Rica: EUNED

Palma, Diego. (1992). La sistematización como estrategia de conocimiento de conocimiento en la educación popular. El estado de la cuestión en América Latina. Papeles del CEAAL, $n^{\circ} 3$. Santiago de Chile.

Paredes Ortiz, Jesús (2003). Juego, luego soy: Teoría de la Actividad Lúdica. Sevilla: Wanceulen Editorial.

Pérez-Yglesias, M. (2012). Estrategia lúdico-creativa: $\mathrm{Al}$ conocimiento y la educación por el placer. Revista Educación, 34 (1), 55-72. Recuperado de https://revistas.ucr.ac.cr/index.php/educacion/issue/ view/160

Prieto Rodríguez, Adriana y Naranjo Polanía, Sandra Patricia. (2005). Cuerpo y movimiento: Perspectivas. Bogotá: Centro Editorial Universidad del Rosario. ISBN 9588225639, 9789588225630
Sánchez Upegui, A. A. (2012). El artículo sistematización de experiencias: construcción de sentido desde una perspectiva crítica. Revista Virtual Universidad Católica Del Norte, (29), 1-7. Recuperado de www.redalyc.org/articulo. oa? id=1942/194214466001

Sousa Santos, B. (2007). La universidad en el siglo XXI: Para una reforma democrática y emancipadora de la universidad. La Paz, Bolivia. Plural Editores.

Subcomisión Lúdico Creativa. (2016). Informe anual de labores 2016. Costa Rica: Consejo Nacional de Rectores (CONARE).

Subcomisión Lúdico Creativa. (2016). Informe quinquenal de la Subcomisión Lúdico Creativa (años 2011 al 2015). Costa Rica: Consejo Nacional de Rectores (CONARE).

Subcomisión Lúdico Creativa. (2017). Informe anual de labores 2017. Costa Rica: Consejo Nacional de Rectores (CONARE).

Subcomisión Lúdico Creativa. (2018). Informe anual de labores 2018. Costa Rica: Consejo Nacional de Rectores (CONARE).

Subcomisión Lúdico Creativa. (2019). Informe anual de labores 2019. Costa Rica: Consejo Nacional de Rectores (CONARE).

Subcomisión Lúdico Creativa. (2017). Informe anual de labores 2017 de la Subcomisión Lúdico Creativa. Costa Rica: Consejo Nacional de Rectores (CONARE). 